\title{
CODES OF ETHICS ARTIFACTS IN AUSTRALIA, CANADA AND SWEDEN: A LONGITUDINAL STUDY
}

\author{
Michael Callaghan, Deakin University, Australia \\ Greg Wood, Deakin University, Australia \\ Göran Svensson, Oslo School of Management, Norway \\ Jang Singh, University of Windsor, Canada \\ Svante Andersson, Halmstad University, Sweden
}

\begin{abstract}
ABSTACT
Based on the Partnership Model of Corporate Ethics (Wood 2002), this longitudinal study examined the measures in place to communicate the ethos of the corporate codes of ethics to internal stakeholders in three countries: Australia, Canada and Sweden. This paper reports the comparative codification of ethics amongst the top companies in these countries over three time periods: 2001-2002, 2005-2006 and 2010-2011.
\end{abstract}

\section{METHODOLOGY}

Questionnaires that were non-sponsored and unsolicited were sent to the top companies (based on revenue) operating in the private sectors within Australia, Canada and Sweden. Companies were asked to answer up to thirty questions. The response rate for each country was (2001-2/2005-6/2010-11): Australia 173/500 (81 indicating that they did have a code), 111/500 (76 codes), and 106/500 (101 codes); Canada 140/500 (100 codes), 106/500 (103 codes), and 91/500 (90 codes); Sweden 72/100 (40 codes), 186/500 (113 codes), and 123/500 (102 codes). The sample taken in Sweden in 2001-2002 differed in size as it was based on a judgmental selection procedure of the top 100 private sector companies. The sample frame and size was extended from stage two in order to facilitate direct comparisons across the three cultures. The results will be reported

\section{RESULTS}

The empirical findings are summarized in the form of: (2001-2/2005-6/2010-11) for each of the Ethics Artifacts measured by the study. Consequences for a Breach of the code are high in each of the countries across all three studies Australia: 89.6\%/95.9\%/98.0\%; Canada: 97.0\%/99.0\%/98.6\%; Sweden: 79.5\%/82.1\%/85.3\%). Ethical Performance Appraisal is more frequent in Australia $(70.5 \% / 77.3 \% / 81.2 \%)$ with a gradual increasing trend in its use that is significantly higher $(\mathrm{p}<.05)$ than both Canada (63.3\%/63.4\%/56.2\%) and Sweden (39.5\%/44.2\%/52\%). The Conduct of Ethical Audits appears to have peaked across the three groups with Australia (23.7\%/49.3\%/50.5\%) and Sweden (62.5/55.1\%/54.5\%) effectively plateauing, and Canada $(40.6 \% / 56.6 \% / 46.5 \%)$ showing decline (not significant at $\mathrm{p}<.05)$ trend. Support of Whistle blowers is more frequent in Australia (43.6\%/84.2\%/88.1\%) and Canada (48.0\%/89.2\%/89.8\%) with Sweden indicating significantly lower $(\mathrm{p}<.05)$ support $(28.2 \% / 43.8 \% / 65.7 \%)$. The use of an Ethics Ombudsman is much more frequent in Canada (55.1\%/66.0\%/59.1\%)than in Australian (32.9\%/31.1\%/52.5\%) and Swedish (33.3\%/34.5\%/42.6\%) organizations. Use of the code as a Guide to Strategic Planning is more frequent in Sweden $(56.4 \% / 60.0 \% / 59.4 \%)$ than in Australia $(43.6 \% / 49.3 \% / 55.4 \%)$ and Canada $(35.7 \% / 39.0 \% / 47.7 \%)$. Ethics Committees were more frequent in Sweden $(50.0 \% / 30.8 \% / 44.6 \%)$ than in Australia $(26.6 \% / 43.8 \% / 52.5 \%)$ and Canada $(38.8 \% / 56.4 \% / 60.2 \%)$ in the 2001-2002 iteration of the study, but it is now more frequent in Canada (60.2\%) than Australia (52.5\%) and Sweden (44.6\%). Use of an Ethics Training Committee is not frequent in any of the three countries across the three studies - Australia $(19.0 \% / 24.7 \% / 36.6 \%)$, Canada $(27.6 \% / 30.0 \% / 39.8 \%)$ and Sweden (17.9\%/19.5\%/45.5\%). Staff Training in Ethics has increased significantly $(\mathrm{p}<.05)$ in the latest iteration of the study with all three countries indicating significant increased use - Sweden (55.0\%/52.2\%/82.2\%), Australia (44.3\%/52.7\%/80.2\%) and Canada (45.8\%/58.4\%/71.6\%).

\section{CONCLUSION}

There now appears to be a general convergence across all three countries toward similar levels of the application and the use of ethical artifacts and progression toward what we would consider to be "best practice". The only significant differences $(\mathrm{p}<.05)$ exhibited between the three countries, as of this latest iteration, are in "consequences for breaching the code" and "whistle blower protection" where Sweden is significantly lower than both Australia and Canada, and "ethical performance appraisal" where Australia indicates significantly higher use. Overall, we are pleased to report that, based on this latest data, all three countries appear to be headed toward world's best practice with regard to the artifacts related to their codes of ethics.

References available on request. 